\title{
Circular chromatic number and Mycielski construction
}

\author{
Hossein Hajiabolhassan ${ }^{1,2}$ \\ and \\ Xuding Zhu ${ }^{1, *}$ \\ ${ }^{1}$ Department of Applied Mathematics \\ National Sun Yat-sen University \\ Kaohsiung 80424, Taiwan \\ ${ }^{2}$ Institute for Studies in Theoretical \\ Physics and Mathematics(IPM) \\ P.O. Box 19395-5746, Tehran, Iran
}

Jan, 2001

\begin{abstract}
This paper gives a sufficient condition for a graph $G$ to have its circular chromatic number equal its chromatic number. By using this result, we prove that for any integer $t \geq 1$, there exists an integer $n$ such that for all $k \geq n$ $\chi_{c}\left(M^{t}\left(K_{k}\right)\right)=\chi\left(M^{t}\left(K_{k}\right)\right)$.
\end{abstract}

Keywords: Circular chromatic number, Mycielski's graphs, chromatic number.

\section{Introduction}

All graphs considered in this paper are finite and simple. Suppose $G=(V, E)$ is a graph and $k \geq 2 d$ are positive integers. A $(k, d)$-colouring of $G$ is a mapping

*Supported in part by the National Science Council under grant NSC91-2115-M-110-003. 
$c: V \rightarrow\{0,1, \cdots, k-1\}$ such that for any edge $x y$ of $G, d \leq|c(x)-c(y)| \leq k-d$. The circular chromatic number $\chi_{c}(G)$ of $G$ is defined by

$$
\chi_{c}(G)=\inf \{k / d \text { : there exists a }(k, d) \text {-colouring of } G\} \text {. }
$$

The circular chromatic number (also known as the star chromatic number) is a natural generalization of the chromatic number (note that a $(k, 1)$-colouring is simply a $k$-colouring), and has been studied extensively in the past decade, $[1,2,4,6,11$, $12,13,14,15,16]$. It is known $[12]$ that $\chi(G)-1<\chi_{c}(G) \leq \chi(G)$ for any graph $G$, and there are graphs $G$ with $\chi_{c}(G)=\chi(G)$ as well as graphs with $\chi_{c}(G)$ arbitrarily close to $\chi(G)-1$. The question of which graphs $G$ have $\chi_{c}(G)=\chi(G)$ has attracted some attention $[1,4,6,11,12,13,14,15]$. It is NP-hard to determine if a given graph $G$ has $\chi_{c}(G)=\chi(G)[6]$. However, some sufficient conditions for graphs to have $\chi_{c}(G)=\chi(G)$ can be found in the literature $[1,4,6,11,15,13]$.

This paper gives another sufficient condition for graphs to have $\chi_{c}(G)=\chi(G)$. This condition is then applied to the study of the circular chromatic number of Mycielski's graphs, especially, the circular chromatic number of the iterated Mycielskian of complete graphs.

For a graph $G$ with vertex set $V(G)=V$ and edge set $E(G)=E$, the Mycielskian $M(G)$ of $G$ is the graph with vertex set $V \cup V^{\prime} \cup\{u\}$, where $V^{\prime}=\left\{x^{\prime}: x \in V\right\}$, and edge set $E \cup\left\{x^{\prime} y: x y \in E\right\} \cup\left\{x^{\prime} u: x^{\prime} \in V^{\prime}\right\}$. The vertex $x^{\prime}$ is called the twin of the vertex $x$ (and $x$ is also called the twin of $x^{\prime}$ ); and the vertex $u$ is called the root of $M(G)$. If there is no ambiguity we shall always use $u$ as the root of $M(G)$. For $t \geq 2$, let $M^{t}(G)=M\left(M^{t-1}(G)\right)$.

There is a very simple formula for $\chi(M(G))$ in terms of $\chi(G)$, i.e., $\chi(M(G))=$ $\chi(G)+1[10]$, as well as a very simple formula for $\chi_{f}(M(G))$ in terms of $\chi_{f}(G)$, i.e., $\chi_{f}(M(G))=\chi_{f}(G)+1 / \chi_{f}(G)[8] .\left(\chi_{f}(G)\right.$ denotes the fractional chromatic number of $G)$. However, there is no simple formula for $\chi_{c}(M(G))$ in terms of $\chi_{c}(G)$.

The problem of calculating the circular chromatic number of Mycielski's graphs has been investigated in $[4,3,7]$. It turns out that the circular chromatic number of $M(G)$ is not determined by the circular chromatic number of $G$ alone. Rather, it depends on the structure of $G$. Even for graphs $G$ with very simple structure, it is still difficult to determine $\chi_{c}(M(G))$. 
The problem of determining the circular chromatic number of the iterated Mycielskian of complete graphs was discussed in [3]. It was conjectured in [3] that if $n \geq t+2 \geq 3$, then $\chi_{c}\left(M^{t}\left(K_{n}\right)\right)=\chi\left(M^{t}\left(K_{n}\right)\right)=n+t$. With complicated arguments, the special cases $t=1,2$ of this conjecture have been proved in [3,4]. A simpler proof of these special cases was given in [4] (for $t=2$, the result proved in [4] is slightly weaker, i.e., it was proved in [4] that for $\left.n \geq 5, \chi_{c}\left(M^{2}\left(K_{n}\right)\right)=\chi\left(M^{2}\left(K_{n}\right)\right)=n+2\right)$.

We shall prove in this paper that for any integer $t \geq 1$, if $n \geq 2^{t}+2$ then $\chi_{c}\left(M^{t}\left(K_{n}\right)\right)=\chi\left(M^{t}\left(K_{n}\right)\right)=n+t$.

\section{A sufficient condition for $\chi_{c}(G)=\chi(G)$}

For $k \geq 2 d$ and $\operatorname{gcd}(k, d)=1$, let $G_{d}^{k}$ be the graph with vertex set $\{0,1, \cdots, k-1\}$ and in which $j \sim j^{\prime}$ if and only if $d \leq\left|j-j^{\prime}\right| \leq k-d$. A homomorphism from a graph $G=(V, E)$ to a graph $G^{\prime}=\left(V^{\prime}, E^{\prime}\right)$ is a mapping $f: V \rightarrow V^{\prime}$ such that $f(x) f(y) \in E^{\prime}$ whenever $x y \in E$. It is well known [16] and easy to see that if $G$ admits a homomorphism to $G^{\prime}$ then $\chi_{c}(G) \leq \chi_{c}\left(G^{\prime}\right)$. It follows from the definition that a $(k, d)$-colouring of a graph $G$ is simply a homomorphism from $G$ to $G_{d}^{k}$. When considering a $(k, d)$-colouring of $G$ (or equivalently, a homomorphism from $G$ to $G_{d}^{k}$ ), we view the colours $0,1, \cdots, k-1$ as cyclically ordered. For $a, b \in\{0,1, \cdots, k-1\}$, the interval $[a, b]_{k}$ means the interval from $a$ to $b$ in this cyclic order. For example, $[2,5]_{k}=\{2,3,4,5\}$ and $[5,2]_{k}=\{5,6, \cdots, k-1,0,1,2\}$. The following lemma is an easy consequence of a result of [13].

Lemma 1 Suppose $G=(V, E)$ is a graph with $\chi_{c}(G)=k / d$ and that $c: V \rightarrow$ $\{0,1, \cdots, k-1\}$ is $a(k, d)$-colouring of $G$. Then for each $i \in\{0,1, \cdots, k-1\}$, there exists a vertex $x$ with $c(x)=i$ which is adjacent to a vertex $y$ with $c(y)=i+d$. Here the addition is modulo $k$.

Proof. Assume to the contrary that there exists an index $i$ such that no vertex of colour $i$ is adjacent to a vertex of colour $i+d$. Let $e=i(i+d)$. Then the colouring $c$ is a homomorphism from $G$ to $G_{d}^{k}-e$. However, it was proved in [13] that $\chi_{c}\left(G_{d}^{k}-e\right)<k / d$, which implies that $\chi_{c}(G) \leq \chi_{c}\left(G_{d}^{k}-e\right)<k / d$, contrary to our assumption. 
Suppose $G=(V, E)$ is a graph. For a vertex $x$ of $G$, we denote by $\bar{N}_{G}(x)$ the set of non-neighbours of $x$, i.e., $\bar{N}_{G}(x)=\{y: y \neq x, y \neq x\}$. For $X \subseteq V$, let $\bar{N}_{G}(X)=\cup_{x \in X} \bar{N}_{G}(x)$. With an abuse of notation, for any subset $X$ of $V$, we shall also use $X$ to denote the subgraph of $G$ induced by $X$. We say $Y \subseteq \bar{N}_{G}(X)$ is a pointwise-dominating set of $\bar{N}_{G}(X)$ if for each $x \in X, \bar{N}_{G}(x)-Y$ is an independent set. We define a parameter $\beta_{G}(X)$ as follows:

$$
\beta_{G}(X)=\min \left\{|Y|: Y \text { is a pointwise-dominating set of } \bar{N}_{G}(X)\right\}
$$

Theorem 2 Suppose $G=(V, E)$ is a graph and $X$ is a clique of $G$. If $\chi_{c}(G)=k / d$ (with $\operatorname{gcd}(k, d)=1)$, then

$$
|X|(d-1) \leq 2 \beta_{G}(X)
$$

Proof. Let $c: V \rightarrow\{0,1, \cdots, k-1\}$ be a $(k, d)$-colouring of $G$. It is well known $[2,12]$ (and also follows from Lemma 1 ) that $c$ must use every colour. Assume that $X=\left\{x_{1}, x_{2}, \cdots, x_{m}\right\}$ and $c\left(x_{i}\right)<c\left(x_{i+1}\right)$.

For $i=0,1, \cdots, k-1$, let $A_{i}=c^{-1}(i)$, i.e., $A_{i}$ is the set of vertices coloured by colour $i$. In the following, all the additions and subtractions of the indices are carried out modulo $k$.

Let $Y$ be a pointwise-dominating set of $\bar{N}_{G}(X)$ with $|Y|=\beta_{G}(X)$.

Construct a bipartite graph $H$ with $X, Y$ as its two parts so that $x_{i} \sim y$ if and only if

$$
c(y) \in\left[c\left(x_{i}\right)-d+1, c\left(x_{i}\right)+d-1\right]_{k}-\left\{c\left(x_{i}\right)\right\} .
$$

We shall show that each vertex of $X$ has degree at least $d-1$ in $H$ and each vertex of $Y$ has degree at most 2 in $H$.

Suppose $x_{i} \in X$ and $c\left(x_{i}\right)=a$. For each $\ell \in\{1,2, \cdots, d-1\}, A_{a-d+\ell} \cup A_{a+\ell} \subseteq$ $\bar{N}_{G}\left(x_{i}\right)$. By Lemma 1 , there exists an edge joining a vertex of $A_{a-d+\ell}$ to a vertex of $A_{a+\ell}$. Since $\bar{N}_{G}\left(x_{i}\right)-Y$ is an independent set, we conclude that $A_{a-d+\ell} \cup A_{a+\ell}$ contains at least one vertex of $Y$. It follows that $x_{i}$ has degree at least $d-1$ in $H$. 
Suppose $y \in Y$ and $c(y) \in\left[c\left(x_{i}\right), c\left(x_{i+1}\right)\right]_{k}$. Since $X$ is a clique, we know that for any $x_{j} \in X$ where $j \neq i, i+1$,

$$
\left[c\left(x_{j}\right)-d+1, c\left(x_{j}\right)+d-1\right]_{k} \cap\left[c\left(x_{i}\right), c\left(x_{i+1}\right)\right]_{k}=\varnothing .
$$

Therefore $y \nsim x_{j}$ in $H$. So $y$ has degree at most 2 in $H$. Hence

$$
|X|(d-1) \leq|E(H)| \leq 2|Y|
$$

and the proof is complete.

Corollary 3 If $G$ has a clique $X$ such that $|X|>2 \beta_{G}(X)$, then $\chi_{c}(G)=\chi(G)$.

Proof. Otherwise $\chi_{c}(G)=k / d$ for some $k, d$ with $\operatorname{gcd}(k, d)=1$ and $d \geq 2$. By Theorem 2, we have $|X| \leq|X|(d-1) \leq 2 \beta_{G}(X)$, contrary to our assumption.

Corollary 3 can be generalized to the following:

Theorem 4 Suppose $G=(V, E)$ is a graph. If there exists a subset $X \subseteq V$ such that $\chi(X)>2 \beta_{G}(X)$, then $\chi_{c}(G)=\chi(G)$.

Proof. Assume to the contrary that $\chi_{c}(G)=k / d$ for some $d \geq 2($ and $(k, d)=1)$. Let $c$ be a $(k, d)$-colouring of $G$. We choose a sequence $\left\{x_{1}, x_{2}, \cdots, x_{s}\right\}$ as follows: Let $x_{1} \in X$ be a vertex for which $c\left(x_{1}\right)=\min \{c(x): x \in X\}$. Suppose $x_{i} \in X$ has been chosen. If there is a vertex $x \in X$ with $c(x) \geq c\left(x_{i}\right)+d$, then let $x_{i+1} \in X$ be a vertex for which $c\left(x_{i+1}\right)=\min \left\{c(x): x \in X, c(x) \geq c\left(x_{i}\right)+d\right\}$. Otherwise, let $i=s$ and the construction of the sequence is completed. Let $f: X \rightarrow\{1,2, \cdots, s\}$ be defined as $f(x)=i$ if $c\left(x_{i}\right) \leq c(x)<c\left(x_{i}\right)+d$. Then $f$ is a proper colouring of $X$. Therefore $s \geq \chi(X)$.

The remaining part is similar to the proof of Theorem 2 , with $x_{1}, x_{2}, \cdots, x_{s}$ playing the roles of the vertices of the clique. In case $d=2$, the argument is exactly the same. In case $d \geq 3$, we need to be careful, because in the bipartite graph $H$ constructed in the proof of Theorem 2, those vertices of $Y$ whose colours lie in the interval $\left[c\left(x_{s-1}\right), c\left(x_{s}\right)\right]_{k}$ and $\left[c\left(x_{1}, c\left(x_{2}\right)\right]_{k}\right.$ may have degree greater than 2 . So we do not have $\chi(X)(d-1) \leq 2 \beta_{G}(X)$, but it is easy to show that $\chi(X) \leq 2 \beta_{G}(X)$, contrary to our assumption. The argument is similar, and we omit the details. 


\section{Mycielski's graphs}

Suppose $G=(V, E)$ is a graph and $M(G)=\left(V \cup V^{\prime} \cup\{u\}, E^{\prime}\right)$ is the Mycielskian of $G$. If $X$ is a subset of $V$, then we also consider $X$ as a subset of $V(M(G))$ (because $V$ is a subset of $V(M(G)))$. Recall that for $x \in V$, the twin of $x$ is denoted by $x^{\prime}$. For a subset $X$ of $V$, we shall denote by $X^{\prime}$ the set of twins of vertices in $X$, i.e., $X^{\prime}=\left\{x^{\prime}: x \in X\right\}$.

Lemma 5 Let $M(G)$ be the Mycielskian of $G$. Then for any subset $X$ of $V(G)$, $\beta_{M(G)}(X) \leq 2 \beta_{G}(X)+1$.

Proof. It is easy to see that for any vertex $x$, if $S=\bar{N}_{G}(x)$, then $\bar{N}_{M(G)}(x)=$ $S \cup S^{\prime} \cup\left\{x^{\prime}, u\right\}$. Let $Z=\bar{N}_{G}(X)$. Then

$$
\bar{N}_{M(G)}(X)=Z \cup Z^{\prime} \cup X^{\prime} \cup\{u\} .
$$

Let $Y$ be a pointwise-dominating set of $\bar{N}_{G}(X)$ with $|Y|=\beta(X)$. To complete the proof of Lemma 5, it suffices to show that $Y \cup Y^{\prime} \cup\{u\}$ is a pointwise-dominating set of $\bar{N}_{M(G)}(X)$.

Let $x \in X$ and $a, b \in \bar{N}_{M(G)}(x)-\left(Y \cup Y^{\prime} \cup\{u\}\right)$. We need to prove that $a \not b$. Note that $\bar{N}_{M(G)}(x) \subseteq Z \cup Z^{\prime} \cup\left\{x^{\prime}, u\right\}$. Assume to the contrary that $a \sim b$. Since $Z^{\prime} \cup\left\{x^{\prime}\right\}$ is an independent set, we have $\{a, b\} \nsubseteq Z^{\prime} \cup\left\{x^{\prime}\right\}$. Since $Y$ is a pointwisedominating set of $\bar{N}_{G}(X)$, it follows that $\{a, b\} \nsubseteq Z$. Thus we may assume that $a \in Z-Y$ and $b \in\left(Z^{\prime} \cup\left\{x^{\prime}\right\}\right)-Y^{\prime}$. But $x^{\prime}$ is not adjacent to any vertex of $\bar{N}_{G}(x)$. Therefore $b \neq x^{\prime}$. Assume that $b=c^{\prime}$. Then $c \in Z-Y$. Now $a \sim c^{\prime}$ implies that $a \sim c$, contrary to the assumption that $Y$ a pointwise-dominating set of $\bar{N}_{G}(X)$.

Corollary 6 Suppose $G$ is a graph and $X$ is a subset of $V(G)$. Then for $t \geq 1$,

$$
\beta_{M^{t}(G)}(X) \leq 2^{t} \beta_{G}(X)+2^{t}-1
$$

Proof. By Lemma $5, \beta_{M^{t}(G)}(X) \leq 2\left(\beta_{M^{t-1}(G)}(X)\right)+1$. The conclusion follows by induction. 
Corollary 7 Suppose $G$ is a graph, $t \geq 1$ and $\chi_{c}\left(M^{t}(G)\right)=k / d$ (where $\operatorname{gcd}(k, d)=$ 1). If $X$ is a clique of $G$ then

$$
|X|(d-1) \leq 2^{t+1} \beta_{G}(X)+2^{t+1}-2 .
$$

Corollary 8 If $G$ has a clique $X$ such that $|X| \geq 2^{t+1} \beta_{G}(X)+2^{t+1}-1$, then $\chi_{c}\left(M^{t}(G)\right)=\chi\left(M^{t}(G)\right)$.

In Corollary 8, the clique $X$ can be replaced by any subgraph $X$ of $G$, with $|X|$ be replaced by $\chi(X)$ (see Theorem 4 ).

Corollary 9 Let $G$ be a graph on $n$ vertices and $X$ the set of vertices of degree $n-1$. If $|X| \geq 2^{t+1}-1$, then $\chi_{c}\left(M^{t}(G)\right)=\chi\left(M^{t}(G)\right)$.

Proof. Since each vertex of $X$ has degree $n-1$, it follows that $\bar{N}_{G}(X)=\varnothing$. So $X$ is a clique with $\beta_{G}(X)=0$. The conclusion follows from Corollary 8 .

The special case $t=1$ of Corollary 9 was proved in [4].

Corollary 10 If $n \geq 2^{t+1}-1$, then $\chi_{c}\left(M^{t}\left(K_{n}\right)\right)=\chi\left(M^{t}\left(K_{n}\right)\right)$.

\section{Some improvements}

For the circular chromatic number of the iterated Mycielskian of complete graphs, the following was conjectured in [3]:

Conjecture 1 [3] If $n \geq t+2$, then $\chi_{c}\left(M^{t}\left(K_{n}\right)\right)=\chi\left(M^{t}\left(K_{n}\right)\right)$.

For any integer $t \geq 1$, let $n(t)$ be the minimum integer such that for any $n \geq n(t)$, $\chi_{c}\left(M^{t}\left(K_{n}\right)\right)=\chi\left(M^{t}\left(K_{n}\right)\right)$. Corollary 10 shows that for any $t \geq 1$, the integer $n(t)$ exists and $n(t) \leq 2^{t+1}-1$. It was shown in [3] that $n(t) \geq t+2$. Therefore we have

$$
t+2 \leq n(t) \leq 2^{t+1}-1
$$

Conjecture 1 above is equivalent to saying that $n(t)=t+2$. 
For $t=1$, the upper and lower bound for $n(t)$ coincide and we have $n(1)=3$. For $t=2$, Conjecture 1 was proved in [3]. Hence $n(2)=4$. For $t \geq 3$, there is a big gap between the upper and lower bounds for $n(t)$. In this section, we shall slightly improve the upper bound for $n(t)$.

In Theorem 2, the cardinality of a minimum pointwise-dominating set $Y$ of $\bar{N}_{G}(X)$ is used to bound the cardinality of $X$. A careful analysis of the proof of Theorem 2 shows that what really matters is the number of colour classes of a $(k, d)$ colouring of $G$ that contain elements of $Y$. By finding a $(k, d)$-colouring that colours some pairs of elements of $Y$ with the same colours, we prove the following result, which is a strengthening of Theorem 2 .

Theorem 11 Suppose $G$ is a graph and $X$ is a clique of $G$. If $\chi_{c}(M(G))=k / d$ (where $\operatorname{gcd}(k, d)=1)$, then

$$
(|X|-3)(d-1) \leq 2 \beta_{G}(X)
$$

Proof. Let $Y$ be a pointwise-dominating set of $\bar{N}_{G}(X)$ with $|Y|=\beta_{G}(X)$. By the proof of Lemma 5, we know that $D=Y \cup Y^{\prime} \cup\{u\}$ is a pointwise-dominating set of $\bar{N}_{M(G)}(X)$.

The remainder of the proof is similar to that of Theorem 2. However, instead of the cardinality of $D$, we count the number of colour classes that contain an element of $D$ in a $(k, d)$-colouring of $M(G)$.

Let $f$ be a $(k, d)$-colouring of $M(G)$. We construct a new $(k, d)$-colouring $f^{\prime}$ of $M(G)$ as follows:

$$
\begin{aligned}
& f^{\prime}(x)=f(x) \text { if } x \notin Y^{\prime} \text { or } x=v^{\prime} \in Y^{\prime} \text { and } f(v) \in[f(u)-d+1, f(u)+d-1]_{k} ; \\
& f^{\prime}\left(v^{\prime}\right)=f(v) \text { if } v^{\prime} \in Y^{\prime} \text { and } f(v) \notin[f(u)-d+1, f(u)+d-1]_{k} .
\end{aligned}
$$

It is easy to verify that $f^{\prime}$ is still a $(k, d)$-colouring of $M(G)$. By the definition, there are at most $\beta_{G}(X)$ colour classes $\left(f^{\prime}\right)^{-1}(i)$ such that $i \notin[f(u)-d+1, f(u)+d-1]_{k}$ and $\left(f^{\prime}\right)^{-1}(i)$ contains an element of $D$.

Let

$$
T=\left\{i: 0 \leq i \leq k-1,\left(f^{\prime}\right)^{-1}(i) \cap D \neq \varnothing\right\},
$$


and let

$$
T^{\prime}=T-[f(u)-d+1, f(u)+d-1]_{k} .
$$

The argument above shows that

$$
\left|T^{\prime}\right| \leq \beta_{G}(X)
$$

Now we construct a bipartite graph $H$ with vertex $X \cup T$ and in which $x \in X$ is adjacent to $i \in T$ if $i \in\left[f^{\prime}(x)-d+1, f^{\prime}(x)+d-1\right]_{k}$.

The same argument as in the proof of Theorem 2 shows that each vertex of $X$ has degree at least $d-1$, and each vertex in $T$ has degree at most 2 .

Since $X$ is a clique, the colours of elements of $X$ are far apart from each other (i.e., $f^{\prime}(x) \notin\left[f^{\prime}(y)-d+1, f^{\prime}(y)+d-1\right]_{k}$ if $x, y \in X$ and $x \neq y$ ). Let

$$
X^{\prime}=\left\{x \in X: N_{H}(x) \cap\left[f^{\prime}(u)-d+1, f^{\prime}(u)+d-1\right]_{k} \neq \varnothing .\right.
$$

Easy calculations show that $\left|X^{\prime}\right| \leq 4$.

If $\left|X^{\prime}\right| \leq 3$, then we have

$$
(|X|-3)(d-1) \leq 2 \beta_{G}(X)
$$

If $\left|X^{\prime}\right|=4$, then by calculating the distance between the colours of elements of $X^{\prime}$, one can show that there are two vertices $a, b \in X^{\prime}$ such that

$\left|\left(\left[f^{\prime}(a)-d+1, f^{\prime}(a)+d-1\right]_{k} \cup\left[f^{\prime}(b)-d+1, f^{\prime}(b)+d-1\right]_{k}\right) \cap\left[f^{\prime}(u)-d+1, f^{\prime}(u)+d-1\right]_{k}\right| \leq d-2$.

This would imply that there are at least $d$ edges of $H$ joining $a$ and $b$ to vertices of $T^{\prime}$.

Therefore

$$
2 \beta_{G}(X)-d \geq(|X|-4)(d-1),
$$

which is equivalent to

$$
(|X|-3)(d-1) \leq 2 \beta_{G}(X)-1 .
$$

This completes the proof. 
Corollary 12 Suppose $G$ is a graph, $t \geq 1$ and $\chi_{c}\left(M^{t}(G)\right)=k / d$ (where $\operatorname{gcd}(k, d)=$ 1). If $X$ is a clique of $G$ then

$$
(|X|-3)(d-1) \leq 2^{t} \beta_{G}(X)+2^{t}-2 .
$$

Proof. Note that $M^{t}(G)=M\left(M^{t-1}(G)\right)$ and $X$ is a clique of $M^{t-1}(G)$. By Corollary $6, \beta_{M^{t-1}(G)}(X) \leq 2^{t-1} \beta_{G}(X)+2^{t-1}-1$. The result follows from Theorem 11 .

For $t \geq 2$, the following are improvements of Corollaries 9 and 10, respectively.

Corollary 13 Let $G$ be a graph on $n$ vertices and $X$ the set of vertices of degree $n-1$. If $|X| \geq 2^{t}+2$, then $\chi_{c}\left(M^{t}(G)\right)=\chi\left(M^{t}(G)\right)$.

Corollary 14 If $n \geq 2^{t}+2$, then $\chi_{c}\left(M^{t}\left(K_{n}\right)\right)=\chi\left(M^{t}\left(K_{n}\right)\right)$.

For $t=3$, Corollary 14 asserts that $\chi_{c}\left(M^{3}\left(K_{n}\right)\right)=\chi\left(M^{3}\left(K_{n}\right)\right)$ for $n \geq 10$. Conjecture 1 asserts that $\chi_{c}\left(M^{3}\left(K_{n}\right)\right)=\chi\left(M^{3}\left(K_{n}\right)\right)$ for $n \geq 5$. By pushing further the technique used in the proof of Theorem 11 (with a more complicated argument), one can show that $\chi_{c}\left(M^{3}\left(K_{n}\right)\right)=\chi\left(M^{3}\left(K_{n}\right)\right)$ for $n \geq 8$. It remains unknown if $\chi_{c}\left(M^{3}\left(K_{n}\right)\right)=\chi\left(M^{3}\left(K_{n}\right)\right)$ for $n=5,6,7$.

It was proved in [4] as well as in Section 2 (Corollary 9) that if an $n$-vertex graph $G$ has 3 vertices of degree $n-1$ then $\chi_{c}(M(G))=\chi(M(G))$. The following is an improvement of this.

Theorem 15 Let $G=(V, E)$ be a graph on $n \geq 3$ vertices. If $G$ has 2 vertices of degree $n-1$, then $\chi_{c}(M(G))=\chi(M(G))$.

Proof. Let $a, b$ be two vertices of $G$ of degree $n-1$. Let $a^{\prime}, b^{\prime}$ be the twins of $a, b$, respectively in $M(G)$, and let $u$ be the root of $M(G)$. Assume to the contrary that $\chi_{c}(M(G))=k / d$ for some $d \geq 2$ (and $\operatorname{gcd}(k, d)=1$ ). Let $f$ be a $(k, d)$-colouring of $M(G)$. Assume $f(a)=i$. Then none of $f^{-1}(i-d+1), \cdots, f^{-1}(i+d-1)$ is empty and each contains only vertices that are not adjacent to $a$. However, the only vertices of $M(G)$ not adjacent to $a$ are $a^{\prime}$ and $u$. So we must have $d=2$ and, say, $f^{-1}(i-1)=\left\{a^{\prime}\right\}, f^{-1}(i)=\{a\}$ and $f^{-1}(i+1)=\{u\}$. 
By symmetry, we have $f^{-1}(i+2)=\{b\}$ and $f^{-1}(i+3)=\left\{b^{\prime}\right\}$. Since $n \geq$ 3, $G$ has vertices other than $a$ and $b$. Now for each vertex $x \in V-\{a, b\}$, since $f(x) \notin\{f(u)-1, f(u), f(u)+1\}$, we can assume that $f\left(x^{\prime}\right)=f(x)$ (if $f(x) \neq f\left(x^{\prime}\right)$, we can recolour $x^{\prime}$ with the colour of $x$ to obtain another $(k, d)$-colouring of $\left.M(G)\right)$. Since every vertex of $V-\{b\}$ is adjacent to $b^{\prime}$, and since $f(x)=f\left(x^{\prime}\right)$ for every $x \in V-\{a, b\}$, we conclude that $f^{-1}(i+4)=\varnothing$. But this is contrary to Lemma 1 .

Theorem 15 is sharp in the sense that there are graphs $G$ with one universal vertex for which $\chi_{c}(M(G)) \neq \chi(M(G))$. Let $W_{2 n+1}$ be the odd wheel which is obtained from the odd cycle $C_{2 n+1}$ by adding a universal vertex. Then for $n \geq 2$, it is easy to show that $\chi_{c}\left(M\left(W_{2 n+1}\right)\right) \leq 4.5<\chi\left(M\left(W_{2 n+1}\right)\right)$, and the argument as in the proof of Theorem 15 shows that $\chi_{c}\left(M\left(W_{2 n+1}\right)\right) \geq 4.5$. Hence $\chi_{c}\left(M\left(W_{2 n+1}\right)\right)=4.5$, which was conjectured in [9].

\section{References}

[1] H. L. Abbott and B. Zhou, The star chromatic number of a graph, J. Graph Theory, 17(1993), 349-360.

[2] J. A. Bondy and P. Hell, A note on the star chromatic number, J. Graph Theory, 14(1990), 479-482.

[3] G. J. Chang, L. Huang, and X. Zhu, The circular chromatic number of Mycielski's graphs, Discrete Mathematics, 205(1999), 23-37.

[4] G. Fan, Circular chromatic number and Mycielski graphs, manuscript, 2000.

[5] D. C. Fisher, Fractional colourings with large denominators, J. Graph Theory, 20(1995), 403-409.

[6] D. R. Guichard, Acyclic graph colouring and the complexity of the star chromatic number, J. Graph Theory, 17(1993), 129-134.

[7] L. Huang and G. J. Chang, The circular chromatic number of the Mycielskian of $G_{k}^{d}$, J. Graph Theory, 32(1999), 63-71.

[8] M. Larsen, J. Propp, and D. Ullman, The fractional chromatic number of Mycielski's graphs, J. Graph Theory, 19(1995), 411-416. 
[9] K. W. Lih, L. Tong, and W. Wang, Acyclic orientations and the circular chromatic number of a graph, manuscript.

[10] J. Mycielski, Sur le colouriage des graphes, Colloq. Math., 3(1955), 161-162.

[11] E. Steffen and X. Zhu, On the star chromatic numbers of graphs, Combinatorica, 16(1996), 439-448.

[12] A. Vince, Star chromatic number, J. Graph Theory, 12(1988), 551-559.

[13] X. Zhu, Star chromatic numbers and products of graphs, J. Graph Theory, 16(1992), 557-569.

[14] X. Zhu, Uniquely H-colourable graphs with large girth, J. Graph Theory, 23(1996), 33-41.

[15] X. Zhu, Graphs whose circular chromatic number equal the chromatic number, Combinatorica, 19(1999), 139-149.

[16] X. Zhu, The circular chromatic number, a survey, Discrete Mathematics, 229(2001), 371-410. 